\title{
Trichoniscidae della grotta di su Mannau, Sardegna Sud-Occidentale (Crustacea, Isopoda)
}

\author{
Roberto ARGANO*
}

Sulle pendici del monte Linas, a N-W di Cagliari, in Sardegna, si apre un gran numero di vastissime cavità note da sempre per le preziosissime concrezioni e per i reperti archeologici che hanno fornito. Dal punto di vista biologico c'è una disproporzione tra la vastità e varietà degli ambienti sotterranei e la relativa scarsità di informazioni.

La grotta di su Mannau 97 SA/CA (cfr. Fureddu, 1964), presso Fluminimaggiori, si apre a $210 \mathrm{~m}$ slra, una ventina di metri sopra al Rio omonimo (Zuffa, 1967) e, a tutt'oggi, è stata esplorata per uno sviluppo complessivo di $4050 \mathrm{mt}$, compresi i collegamenti sotterranei con cavità ritenute in precedenza indipendenti (Puddu, in litteris). Le esplorazioni sembra siano cominciate intorno al 1930, ma presero una veste scientifica solo dal 1963, con le esplorazioni del G.S. Piemontese del CAI-Uget di Torino in collaborazione con speleologi sardi, e successivamente di speleologi dell'Iglesiente, di Bologna e, recentemente, dello Speleo Club di Cagliari. E'appunto da quest'ultimo gruppo di speleologi che sono state effettuate raccolte faunistiche nella grotta, condotte in particolare da Sergio Puddu. E' a lui infatti che debbo l'invio di due campionature di Trichoniscidae effettuate nel corso di due diverse esplorazioni.

Si tratta dei primi dati biologici di un certo interesse raccolti nella grotta (ofr. Brignoli, in stampa.). Ma è prevedibile che il fitto reticolo diaclasico, in calcare cambrico, in cui si imposta il complesso di gallerie, saloni e fessurazioni della grotta, con tutta la varietà d'ambienti che offre (cfr. Grimandi, 1968), riservi, a ulteriori indagini, sorprese notevoli in questo campo.

Le conoscenze sui Trichoniscidae della Sardegna sono relativamente scarse. Ne sono una prova queste occasionali campionature di Su Mannau che hanno permesso di conoscere tre nuove specie appartenenti a due generi, oltre che nuovi, assolutamente inattesi per la Sardegna. Un quadro completo delle conoscenze sull'argomento verrà diato al termine di ricerche attualmente in corso.

Nella presente nota viene data la descrizione delle tre specie rinvenute nella grotta di Su Mannau con un cenno alle implicazioni sistematiche e biogeografiche che la loro scoperta comporta.

L'olotipo of e urı paratipo $q$ di ciascuna delle specie descritte viene conservato presso il Museo di S'toria Naturale di Verona, il resto dei paratipi presso l'Istituto di Zoologia dell'Università di Roma.

* Istituto di Zoologia dell'Università di Roma. Direttore: Prof. Pasquale Pasquini 


\section{CATALAUNISCUS HIRUNDINELLA N.SP.}

MATERIALE ESAMINATO: 2 ơ e 3 o $\$$ raccolti il 31.III.1968 da S. Puddu a $300 \mathrm{mt}$ circa dall'ingresso, in un grande salone del ramo sinistro alla base di una larga stalagmite. Resti di carte lasciati da precedenti visitatori della grotta hanno permesso il riunirsi di diversi esemplari della stessa specie in un' unico punto.

DIAGNOSI: un Catalauniscus di medie dimensioni $(5,3 \mathrm{~mm})$ in cui il lobo terminale dell'esopodite del pleopode I del o porta una forte incisione a coda di rondine sul lato esterno.

DESCRIZIONE. Dimensioni del o: lunghezza $5,3 \mathrm{~mm}$; larghezza massima del pereion $2,1 \mathrm{~mm}$; larghezza massima del pleon $1,0 \mathrm{~mm}$.

Occhi e pigmento completamente assenti.

Tegumento fortemente tubercolato. I tubercoli, formati da una larga setola centrale, molto corta, circondata da diverse serie concentriche di scaglie, sono disposti in serie trasversali sul cephalon e sul pereion.

Antennule formate da tre articoli, l'ultimo dei quali, piatto e allungato, porta terminalmente una serie di sei estetaschi bastoncellari. Gli articoli del peduncolo delle antenne sono ornati di forti tubercoli conici formati da gruppi compatti di scaglie. Il flagello, i cui articoli sono poco distinti tra loro, porta tre coppie di setole spatoliformi ed un fitto ciuffo di setole terminali.

Le parti boccali non presentano caratteristiche degne di rilievo. Mandibola sinistra con tre penicillae, mandibola destra con una. Il settimo paio di pereiopodi del ơ non presenta nessuna differenzizione sessuale.

Endopodite del pleopode I del ơ triangolare, tre volte circa più lungo che largo, si assottiglia fortemente all'estremità distale su cui è inserita una lunga setola provvista di barbule. Il lobo terminale dell'esopodite porta una forte incisura a coda di rondine.

Apofisi genitale a margini paralleli mucronata all'apice.

L'esopodite del pleopode II del ờ è molto grande arrivando fino alla metà del secondo articolo dell'endopodite. Quest'ultimo, biarticolato, presenta il secondo articolo, lungo poco più della metà del I, troncato distalmente e con una doppia punta terminale.

AFFINITÀ. C.hirundinella n.sp. presenta affinità con la specie spagnola $C$. espanoli Vandel alla quale somiglia, oltre che per le dimensioni, per la forma dell'eso e dell'endopodite del secondo pleopode e per l'endopodite del pleopode I del o.

DERIVATIO NOMINIS. L'incisione dell'esopodite del pleopode I del $\delta$, carattere diagnostico, ha una vaga forma a coda di rondine; di qui il nome della specie. 


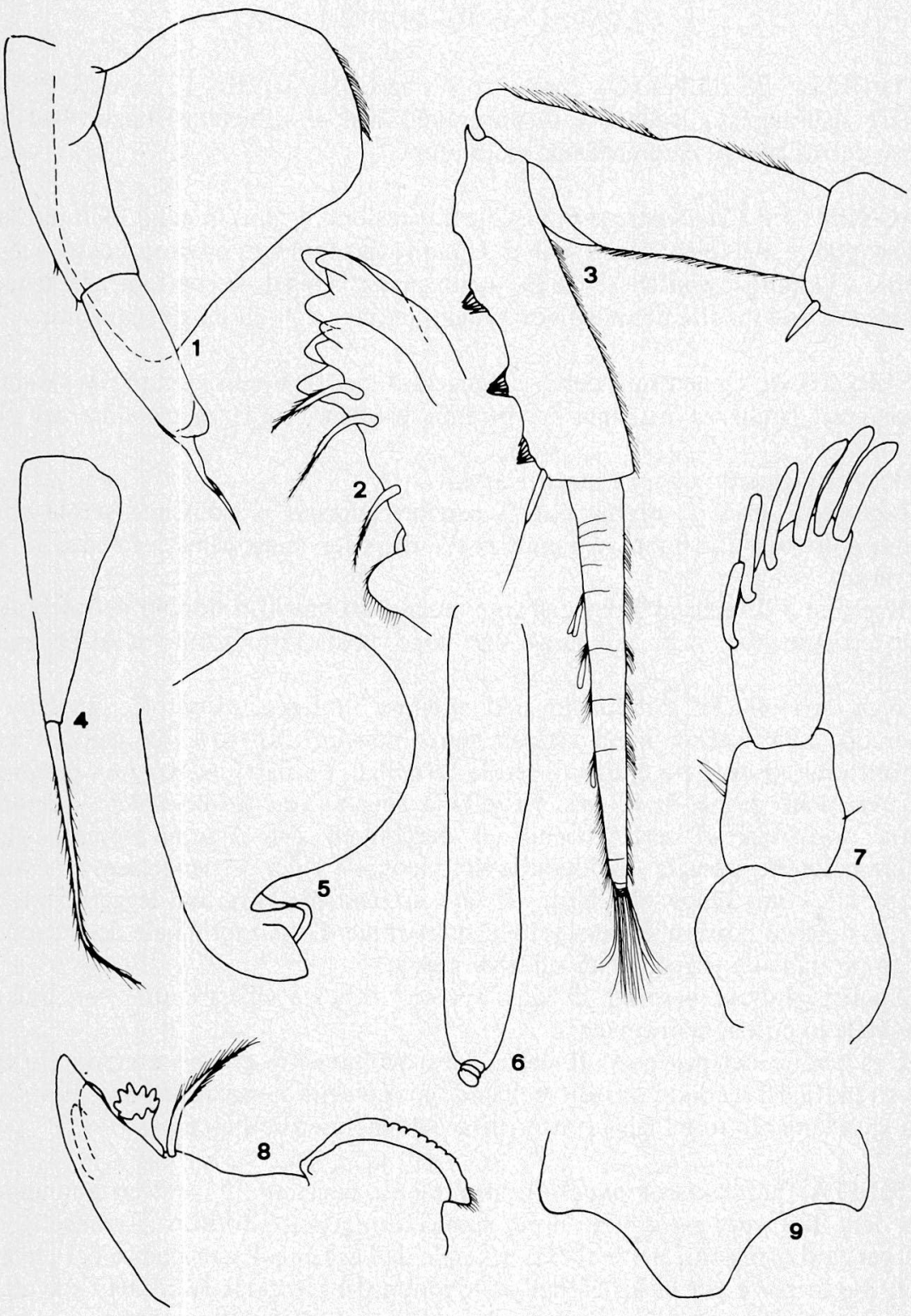

Tav. 1. Catalauniscus hirundinella n.sp.: 1, pleopode II ${ }^{\circ} ; 2$, mandibola sinistra; 3 , antenna; 4,5,6 endopodite, esopodite e apofisi genitale del pleopode I $\delta^{*} ; 7$, antennula; 8 , mandibola destra; 9 , telson. 


\section{CATALAUNISCUS PUDDUI N.SP.}

MATERIALE ESAMINATO: 2 tơ e 5 $९$ raccolti il 3. VIII. 1970 da S. Puddu a $600 \mathrm{mt}$ dall'ingresso, sulle rive di un grande lago di stillicidio (lungh. 40-50mt), presso detriti lasciati da precedenti esploratori.

DIAGNOSI: Un Catalauniscus di piccole dimensioni $(4 \mathrm{~mm})$ in cui il lobo terminale dell'esopodite del pleopode I del ò è molto prominente e arrotondato sul lato esterno. L'apofisi genitale è larga, conformata a spatola, nettamente troncata all'apice. L'endopodite del pleopode II del ơ termina con un lungo filamento.

DESCRIZIONE. Dimensioni del ơ: lunghezza $4 \mathrm{~mm}$ (la 9 può raggiungere i $6 \mathrm{~mm}$ di lunghezza); larghezza massima del pereion 1,4 $\mathrm{mm}$; larghezza massima del pleon $0,8 \mathrm{~mm}$.

Occhi e pigmento completamente assenti.

Tegumenti lisci. Il cephalon e il pereion portano piccolissime setole sottili, visibili solo con un buon ingrandimento, disposte approssimativamente in serie trasversali.

Il primo e il terzo articolo dell'antennula sono lunghi il doppio del secondo. Il terzo articolo, due volte più lungo che largo, porta terminalmente sei bastoncelli sensori.

Sugli articoli del peduncolo dell'antenna, ciuffetti di setole simulano dei tubercoli. Sul flagello, i cui articoli sono indistinti, si trova un paio di setole spatoliformi ed un fitto cuiffo di setole terminali. Le parti boccali non presentano caratteristiche degne di rilievo. Mandibola sinistra con tre penicillae, mandibola destra con una. Il settimo paio di pereiopodi del ot non presenta alcuna differenziazione sessuale. Endopodite del pleopode I del to triangolare, una volta e mezza circa più lungo che largo. Il lato esterno presenta una leggera sinuosità. Terminalmente porta una lunga setola con barbule. Il lobo terminale dell'esopodite, molto prominente, arrotondato sul lato esterno.

Apofisi genitale, a forma di larga spatola troncata all'estremità, con mucrone terminale in posizione mediana.

L'esopodite del pleopode II del ò è particolarmente grande e arriva fin quasi all'estremità del secondo articolo dell'endopodite della stessa appendice, escludendo il lungo filamento terminale caratteristico dell'endopodite stesso.

AFFINITÀ Catalauniscus puddui n.sp. presenta una serie di caratteri comuni con una delle due specie spagnole note: somiglia infatti a C.bolivari (Arcangeli), oltre che per le dimensioni, per la forma generale dell'eso e dell'endopodite del pleopode I del $\delta^{\star}$ e in parte per la forma dell'endopodite del pleopode II. Il fatto che manchi di forti granulazioni sui tegumenti ha importanza relativa esistendo per questo carattere una certa variabilità da una popolazione all'altra, almeno per la specie $C$. espanoli Vandel (Vandel, 1953). L'apofisi genitale ha invece una vaga rassomiglianza con quella di $C$. espanoli. 


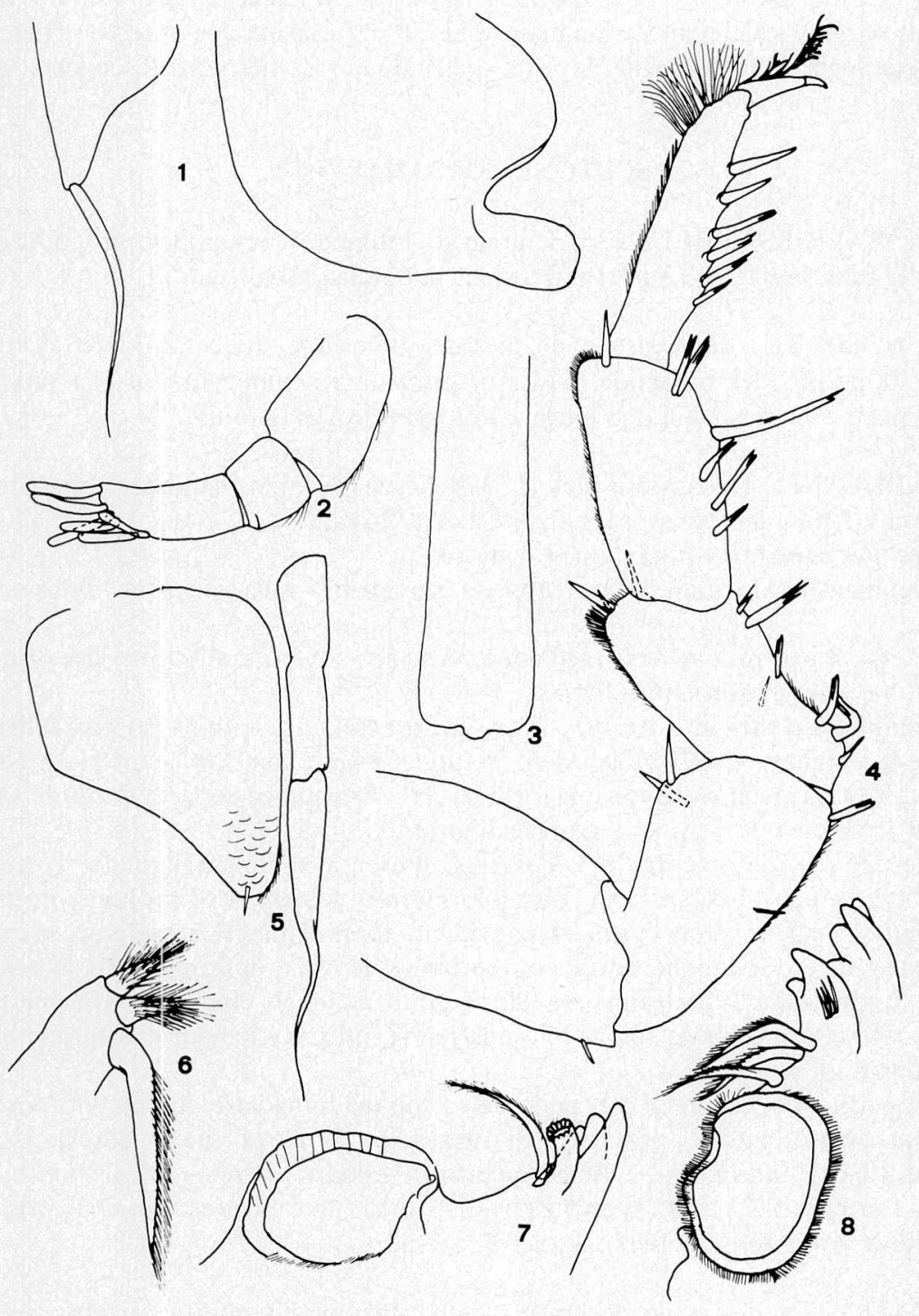

Tav. 2. Catalauniscus puddui n.sp.: 1 , endopodite ed esopodite del pleopode I $\delta^{*} ; 2$, antennula; 3 , apofisi genitale; 4 , pereiopode VII; 5 , pleopode II $\delta ; 6$, endite della maxillula; 7 , mandibola destra; 8 , mandibola sinistra. 
DERIVATIO NOMINIS. La specie è dedicata all'amico Sergio Puddu, che ha raccolto le tre specie di Su Mannau, e al cui entusiasmo costante ed efficiente la biospeleologia della Sardegna deve un gran numero di interessanti scoperte recenti.

\section{SCOTONISCUS JANAS N.SP..}

MATERIALE ESAMINATO: 1 o, privo di antenne, pereiopodi e uropodi, e 2 9o raccolti da S. Puddu il 3.VIII.1970 assieme a Catalauniscus puddui.

DIAGNOSI: Uno Scotoniscus di piccole dimensioni $(3,1 \mathrm{~mm})$ con l'estremità dell'endopodite del pleopode II del ot terminante semplicemente con una punta leggermente ricurva e con una lunga setola provvista di barbule.

DESCRIZIONE. Dimensioni del ${ }^{*}$ : lunghezza $3,1 \mathrm{~mm}$; larghezza massima del pereion 1,2 $\mathrm{mm}$; larghezza massima del pleon $0,6 \mathrm{~mm}$.

Occhi e pigmento completamente assenti.

Tegumento con granulazioni appena percettibili sulla porzione anteriore dei tergiti.

Il terzo ed ultimo articolo dell'antennula, più stretto e allungato dei primi due, porta due estetaschi bastoncellari.

Mandibola destra con tre penicillae, sinistra con due. Lamina esterna della prima mascella con una serie di dieci denti semplici inseriti sul bordo anteriore obliquo; lamina interna terminante con una forte setola tozza conica coperta di setole sottili e due altre setole corte coniche subapicali interne.

L'esopodite del pleopode I del ô si presenta subquadrangolare, fortemente arrotondato agli angoli esterni. L'angolo interno posteriore si prolunga in un lobo allungato piegato verso l'esterno che porta terminalmente una lunga setola con barbule. Altre due lunghe setole con barbule si trovano, internamente, alla base del lobo. Endopodite subrettangolare, due volte più lungo che largo con una forte e lunga setola con barbule sull'angolo posteriore interno e l'angolo posteriore esterno prolungato in un lobo.

Esopodite del secondo pleopode del ot subquadrangolare con angolo posteriore esterno arrotondato e provvisto di una serie di corte setole sottili. Il primo segmento dell'endopodite è lungo quanto l'esopodite. Il secondo segmento, lungo circa il doppio del primo, si assottiglia distalmente terminando con una corta punta ricurva verso l'esterno e una lunga setola sottile.

AFFINITÀ. La posizione geografica della stazione di questa seconda specie di Scotoniscus (tutte le altre entità dello stesso genere, considerate sottospecie della stessa unica specie $S$. macromelos Racovitza, sono descritte per le grotte delle regioni centrali dei Pirenei settentrionali) impone delle considerazioni a livello sistematico. Va sottolineato che si dispone di un unico maschio, per di più mutilato, per quanto tutte le appendici che portano caratteri distintivi siano assolutamente intatte. $\mathrm{Va}$ comunque considerato che eventuali nuovi dati sulla variabilità 


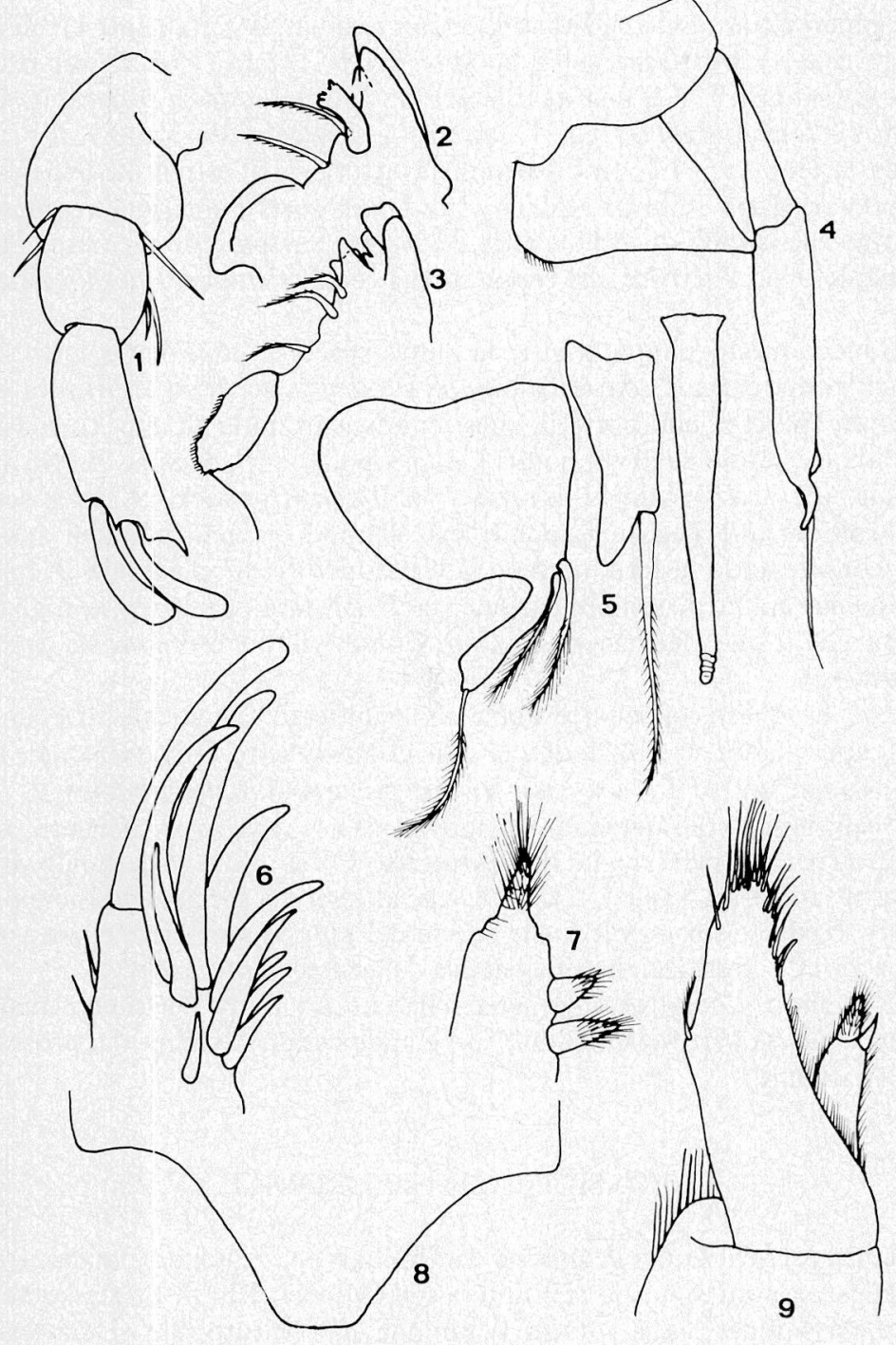

Tav. 3. Scotoniscus janas n.sp.: 1 , antennula; 2,3 , mandibola destra e sinistra; 4 , pleopode II 0 ; 5 , pleopode I $\delta ; 6,7$ maxillula, lamina esterna ed interna; 8 , telson; 9 , massillipede. 
potrebbero portare ad un'analisi diversa.

I due generi Scotoniscus e Trichoniscoides risultano strettamente affini al punto che la presenza di tre setole sull'esopodite del pleopode I ot di Scotoniscus viene considerata (Vandel, 1952) una stabilizzazione di una anomalia riscontrata in alcuni esemplari di Trichoniscoides (in T. mixtus e T. davidi da Vandel 1952 e in $T$. vandeli da Dalens, 1966). Inoltre l'affinità strutturale dell'estremità dell'endopodite del pleopode II ot tra T.davidi e S.m.malarodensis porta a considerare quest'ultima forma come capostipite di tutte le altre del genere Scotoniscus derivante, attraverso una forma del tipo T. davidi, dal ceppo pirenaico di Trichoniscoides (Vandel, 1952, 1960).

Accettando questo punto di vista la nuova specie si inserirebbe sotto il profilo sistematico proprio tra $T$. davidi e S.m.malarodensis acuendo le affinità tra i due generi, tanto che ci si può porre la questione se attribuirla all'uno, considerando la presenza di tre setole sugli esopoditi dei pleopodi I del ot come particolarmente eccezionale (nei casi analoghi osservati in Trichoniscoides la terza setola era presente solo su uno degli endopoditi dei pleopodi I degli individui anomali), o all'altro, considerando la terminazione dell'endopodite del pleopode II del ơ come un eccezionale grado di semplificazione, cioé una fase primitiva, se vogliamo, più vicina alla situazione del genere Trichoniscoides di quanto non sia Scotoniscus m malarodensis.

Il fatto che questa appendice abbia un significato funzionale diverso nei due generi, di apposizione in Scotoniscus e di intromissione in Trichoniscoides ha le sue eccezioni sia nell'uno (T.fouresi) che nell'altro genere (S.m.malarodensis).

In conclusione, considerando la nuova specie come appartenente al genere Scotoniscus,(per la morfologia del pleopode I del $\delta$ ), e accettando il quadro evolutivo proposto da Vandel, S.janas viene ad essere la specie del genere più affine al Trichoniscoides, in particolare alle specie del gruppo pirenaico, e viene a trovarsi alla prima fase differenziazione filogenetica del genere Scotoniscus.

Derivatio nominis: domus de janas, case selle fate, è il nome che si dà attualmente a quelle piccole cavità artificiali scavate con grande cura nelle rocce dai protosardi per custodirvi i defunti.

\section{CONSIDERAZIONI GENERALI}

La geonemia del genere Caralauniscus era limitata alla regione catalana, tra la valle del Llobregat, a nord, e quella dell'Ebro a sud (Vandel, 1953). Lo stesso nome dato al genere da Vandel lascia intuire l'opinione dell'Autore che si trattasse di un fenomeno endemico, cioè che nella differenziazione filogenetica dei generi Catalauniscus, Nesiotoniscus e Balkanoniscus dal ceppo Speleonethes (o forma vicina) (Vandel 1953, 1960) la storia del primo genere fosse limitata ad una regione ristretta. L'esistenza in Sardegna di due specie di questo genere che, per la loro morfologia, ripetono curiosamente la stessa identica situazione che in Spagna (sono infatti sorprendenti le forti rassomiglianze hirundinella-espanoli e puddui-bolivari) riapre naturalmente la questione, o meglio avvalora l'affermazione di Vandel che si 
tratti di un genere tyrrhénien, ma suggerisce cautela per altre conclusioni in attesa di dati che potre əbero chiarire in maniera del tutto imprevedibile i rapporti tra $\mathrm{i}$ generi suelencati.

Un discorso analogo si può fare per la nuova specie di Scotoniscus. Al momento, sia che si considerino affinità con Trichoniscoides (meno impegnative dato il vasto areale del genere) o con Scotoniscus (considerato anche questo un fenomeno endemico del versante nord dei Pirenei centrali) si ripropone solo un'affinità tra la storia faunistica clell'isola e quella dei Pirenei. Si può dire che, pur rispettando il quadro della meccanica di differenziazione suggerito da Vandel (1952), il fenomeno ha interessato un'area più vasta di quella supposta precedentemente ed è da rivedere l'idea che tale differenziazione abbia avuto origine nei Pirenei. S. janas risulta infatti essere la forma più primitiva del gruppo.

\section{RÉSUMÉ}

Description de trois nouvelles espèces anophtalmes et dépigmentées de Trichoniscidae capturées à la grotte de Su Mannau en Sardaigne. Deux d'entre-elles appartiennent au genre Catalauniscus Vandel: Catalauniscus hirundinella n.sp. qui présente quelques affinités avec l'espèce espagnole $C$. espanoli Vandel et Catalauniscus puddui n.sp. qui se râpproche également d'une forme ibérique, $C$. bolivari Arcangeli. La troisième (Scotoniscus janas n.sp.) fait partie du genre endémique Scotoniscus Racovitza, monospécifique jusqu'à ce jour et représenté par une série de sous-espèces des Pirénées septentrionales; toutes très proches de la forme spécifique $S$.macromelos Racovitza.

$\mathrm{La}$ découverte de ces trois nouvelles espèces apportent des arguments supplémentaires ì la thèse d'une origine tyrrhénienne de ces lignées de Trichoniscidae.

\section{SUMMARY}

Description of three eyeless and apigmented new species of Trichoniscidae which have been found in Su Mannau cave in Sardinia. Two of them belong to the genus Catalauniscus Vandel: Catalauniscus hirundinella n.sp. offering few relationships with the spanish species $C$. espanoli Vandel, and Catalauniscus puddui n.sp. which has also similar features to an iberian form, $C$. bolivari Arcangeli. The third (Scotoniscus janas n.sp.) belongs to the endemic genus Scotoniscus Racovitza, until now monospecific and represented by a series of subspecies from the Northern Pyrenees, all nearly related to the specific form $S$. macromelos Racovitza.

The discovery of those three new species brings forward additional arguments to the thesis of a Tyrrhenian origin of these taxa of Trichoniscidae. 


\section{BIBLIOGRAFIA}

BRIGNOLI P.M., in stampa - Catalogo dei ragni cavernicoli italiani.

DALENS, H., 1966 - Une nouvelle espèce endogée de Trichoniscoides, T. vandeli n.sp. provenant du Tarn, (Crustacés; Isopodes terrestres). - Ann. Speleol., 21; 651-653.

FUREDDU P.A., 1964 - Elenco catastale delle grotte della Sardegna-Rass. Spel. It., 15, 3-80. GRIMANDI P., 1968 - "Su Mannau" - Sottoterra - Boll. infG.S.B.-CAI e S.C.B.. Bologna, $20,12-23$.

VANDEL, A., 1952 - Isopodes terrestres (Troisième série). Biospeologica. Arch. Zool. Exp. Gen., 88 221-362.

VANDEL, A., 1953 - Espèces nouvelles ou peu connues de Trichoniscidae cavernicole recueillies en Espagne orientale. Notes Biospeol., 8, 51-66.

VANDEL, A., 1960 - Isopodes terrestres (Première partie). Faune de France, 64, 1-416.

ZUFFA, G.C., 1967 - Grotta di Su Mannau - Sottoterra - Boll. inf. G.S.B.-CAI e S.C.B., Bologna, 16. 